\title{
The XLENS Project: Do More Massive Early-Type Galaxies Have More Dark Matter or Different Stellar IMFs?
}

\author{
Chiara Spiniello \\ Kapteyn Astronomical Institute, University of Groningen, \\ Postbus 800, 9700 AV Groningen, the Netherlands \\ email: spiniello@astro.rug.nl
}

\begin{abstract}
The X-shooter Lens Survey (XLENS) aims to study the interplay of dark matter (DM) and stellar content in the inner regions of massive early-type galaxies (ETGs) by combining strong gravitational lensing, dynamical models, and spectroscopic stellar population analysis. XLENS targets a sample of ETGs from the SLACS survey (The Sloan Lens ACS Survey, e.g. Bolton et al. 2006) with velocity dispersions $\geqslant 250 \mathrm{~km} \mathrm{~s}^{-1}$ using the X-Shooter spectrograph on ESO's Very Large Telescope. Recent observations indicate that the internal dark-matter fraction of ETGs increases rapidly with galaxy mass, although some hints for a varying initial mass function (IMF) have also been suggested, where the low-mass end of the stellar IMF steepens with galaxy mass. XLENS first results unambiguously confirm that DM plays an important role already within one effective radius for very massive systems (Spiniello et al. 2011). Moreover, studying equivalent widths of certain red spectral features which are indicators of low-mass stars in massive ETGs (e.g. $\mathrm{NaI}$ and $\mathrm{TiO} 2$ ) as a function of age and metallicity (i.e. $\mathrm{Mg} b, \mathrm{Fe}, \mathrm{H} \beta$ ), and as function of stellar velocity dispersion, has shown that the IMF slope is varying mildly with galaxy mass (Spiniello et al. 2012).
\end{abstract}

Keywords. dark matter, galaxies, IMF, structure, evolution

\section{Introduction}

The relationship between baryonic matter and dark matter in the internal regions of early-type galaxies (ETGs) is particularly important to comprehend the processes that drive in hierarchical galaxy formation. While stars are supposed to dominate in the innermost region, dark matter - which dominates most of the dynamics during galaxy assembly - is found to play a non-negligible role in the central region, especially for very massive systems. In fact, new observations indicate that the internal DM fraction increases monotonically with the mass of the galaxy (e.g. Auger et al. 2010, Barnabé et al. 2011), assuming a universal IMF.

When constraining the star formation, metallicity and gas/dust content of galaxies, the initial mass function (IMF) is often assumed to be universal and equal to that of the solar neighbourhood (Kroupa 2001; Chabrier 2003; Bastian, Covey \& Meyer 2010). However, evidence has recently emerged that the IMF might evolve (Davé 2008; van Dokkum 2008) or depend on the stellar mass of the system (e.g. Worthey 1992; Trager et al. 2000; Graves et al. 2009; Treu et al. 2010; Auger et al. 2010b; Napolitano 2010; van Dokkum \& Conroy 2010; Spiniello et al. 2012). van Dokkum \& Conroy (2010; hereafter vDC10) suggested that low-mass stars $\left(\leqslant 0.3 M_{\odot}\right)$ could be more prevalent in more massive ETGs. The increase in the mass-to-light ratio (M/L) of galaxies with galaxy mass may thus be partly due to a changing IMF rather than an increasing dark matter fraction, consistent with previous suggestions (Treu et al. 2010, Auger et al. 2011, Barnabè et al. 
2011, Dutton et al. 2012, Cappellari et al. 2012). To assess whether this result is genuine and model-independent, it is of crucial importance to disentangle stellar and dark matter contributions in the inner regions of galaxies, and to calculate stellar M/L values with an accuracy better than $20 \%$ (set by the range in DM fractions). With the XLENS survey we have set out to achieve this ambitious goal in a substantial sample of individual ETGs that have strong gravitational lensing, stellar kinematic and stellar-population information available.

\section{The X-Shooter Lens Survey (XLENS)}

\subsection{A pilot program: The Cosmic Horseshoe}

The first result from the X-shooter Lens Survey (XLENS) is an analysis of the massive ETG SDSS J1148+1930 at redshift $z=0.444$ (Spiniello et al. 2011). We combine its extended kinematic profile - derived from X-shooter spectra - with strong gravitational lensing and multi-color information derived from SDSS images. We calculated the luminosity-weighted stellar velocity dispersion $\left(\left\langle\sigma_{*}\right\rangle\left(\leqslant R_{e f f}\right)=351 \pm 10 \mathrm{~km} \mathrm{~s}^{-1}\right)$ and we obtain a projected stellar mass fraction $\left(f_{*}\left(<R_{\mathrm{E}}\right)=0.19_{-0.09}^{+0.04}\right)$ from a two component mass model of the lens galaxy. From SDSS colors, we obtain a second independent internal stellar mass fraction for different assumed IMFs (i.e. Chabrier, Salpeter, $x=3$, and $x=3.5$, assuming $\left.d N / d M \propto M^{-x}\right)$. We find that the lensing and kinematic constraints on the stellar mass fraction agree well with those independently derived from the SDSS colors for a Salpeter IMF. Dwarf-rich IMFs in the lower mass range of $0.1-0.7 M_{\odot}$, with $x \geqslant 3$ are excluded at the $>90 \%$ C.L. and for $x=3.5$ violate the total lensing-derived mass limit. We conclude that this very massive early-type galaxy is dark-matter dominated inside one effective radius, extending the trend recently found from massive SLACS galaxies (Treu et al. 2009, Auger et al. 2010) and that a very steep IMF can not fully account for this trend.

\subsection{Evidence for a mild steepening and Bottom-heavy IMF in Massive ETGs}

In Spiniello et al. (2012) we investigate possible IMF variations with galaxy mass. We studied equivalent widths (EW) - focussing on two absorption lines (NaI and TiO2) of low-mass stars $\left(\leqslant 0.3 \mathrm{M}_{\odot}\right)$ - for luminous red galaxy spectra from the Sloan Digital Sky Survey (SDSS) and X-Shooter Lens Survey (XLENS). indicators ( $\mathrm{Mg} b, \mathrm{Fe}, \mathrm{H} \beta$ ), of $\mathrm{NaD}$, and of stellar velocity dispersion. We compare the NaI and $\mathrm{TiO} 2 \mathrm{EWs}$ to those derived from simple stellar population models computed for different assumed IMFs, ages, $[\alpha / \mathrm{Fe}]$, and elemental abundances (Fig. 1). We find that current state-of-the-art SSP models are only able to simultaneously reproduce the observed $\mathrm{NaD} \lambda 5895$ and $\mathrm{NaI} \lambda 8190$ features for the lower-mass (around $\sigma_{*}$ ) ETGs but deviate increasingly for more massive ETGs. In particular, the NaD EWs do not follow the models well (Fig. 1(b)). We conclude that the $\mathrm{NaD}$ feature is affected by as-of-yet not understood processes in the more massive ETGs $\left(\sigma>250 \mathrm{~km} \mathrm{~s}^{-1}\right)$. Despite this, we find that the TiO2 $\lambda 6230$ and the NaI $\lambda 8190$ are particularly promising features to decouple the IMF from stellar population, age, metallicity, and abundance pattern, especially when combined with metallicity-dependent indices. We also observe a clear trend of an increasing IMF slope between $\sigma=200-$ $335 \mathrm{~km} \mathrm{~s}^{-1}$ from Salpeter $(x=2.35)$ to $x \approx 3.0$. The XLENS ETG (SDSSJ0912+0029) and one SDSS ETG (SDSSJ0041-0914) appear to require both an extreme dwarf-rich IMF $(x \geqslant 3.0)$ and a high sodium enhancement $([\mathrm{Na} / \mathrm{Fe}]=+0.4)$. However, lensing constraints on the total mass of the XLENS system within its Einstein radius limit a bottom-heavy IMF to a power-law slope of $x \leqslant 3.0$ at the $90 \%$ C.L. (Table 1 ). A full spectral comparison, 

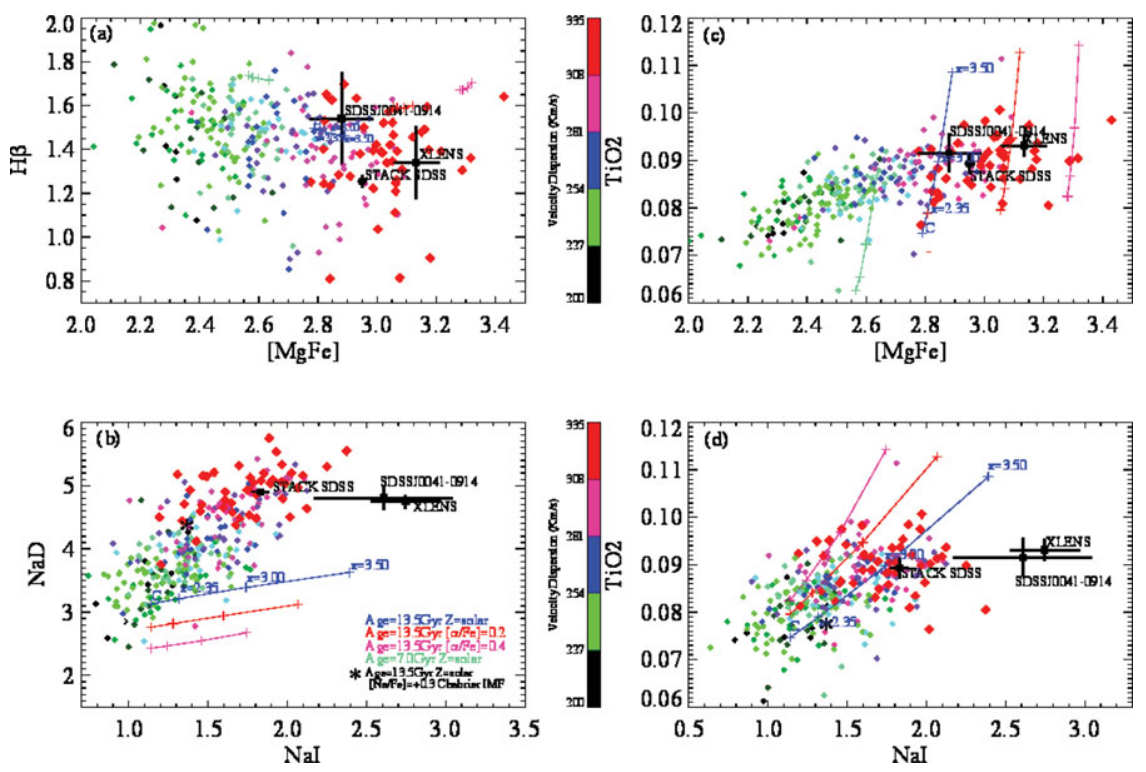

Figure 1. Index-index plots of the main absorption features. Lines and crosses are different SSP models from single population models of Conroy \& van Dokkum 2012 with increasing IMF (from Chabrier, to an extremely dwarf-rich IMF with a slope of $x=3.5$ ). Points coloured according to their velocity dispersions are individual SDSS galaxies, with index errors similar to SDSS J0041-0914. In the plots showing sodium (Panel (b) and Panel (d)), the XLENS system SDSSJ0912+0029 requires a very steep IMF, violating lensing constraints on its total mass. From Panel (c), it is clear that IMF varies with mass: the most massive ETGs require an IMF slope slightly steeper than Salpeter. A Chabrier-type IMF systematically underestimates the SDSS TiO2 EWs.

Table 1. Variation with IMF of M/L and stellar mass fraction within the Einstein radius.

\begin{tabular}{lcccc}
\hline $\begin{array}{l}\text { IMF slope } \\
\left(d N / d m=M^{-x}\right)\end{array}$ & $\begin{array}{c}(M / L)_{D S E P, B}^{*} \\
([\alpha / F e]=0.0)\end{array}$ & $\begin{array}{c}(M / L)_{D S E P, V}^{*} \\
([\alpha / F e]=0.0)\end{array}$ & $f_{B}^{*}(1)$ & $f_{V}^{*}(2)$ \\
\hline-2.35 & $10.2 \pm 3$ & $7.2 \pm 2$ & $0.75 \pm 0.2$ & $0.59 \pm 0.18$ \\
-3.00 & $22 \pm 6$ & $16 \pm 5$ & $1.6 \pm 0.5$ & $1.4 \pm 0.4$ \\
-3.50 & $43 \pm 13$ & $29 \pm 9$ & $2.4 \pm 0.8$ & $2.4 \pm 0.7$ \\
\hline
\end{tabular}

Notes:

${ }^{(1)}: f_{B}^{*}=\left(L_{E i n} / M_{E i n}\right) \times(M / L)_{B}^{*},{ }^{(2)}: f_{V}^{*}=\left(L_{E i n} / M_{E i n}\right) \times(M / L)_{V}^{*}$

Constraints on the mass and luminosity within $M_{E \text { in }}$ from Auger et al. (2009) and Barnabè et al. (2009). All quantities are calculated in the rest-frame $V$ - and $B$-band.

in combination with more detailed lensing and dynamical constraints is planned to assess whether $\mathrm{NaI}$ and $\mathrm{NaD}$ (in some instances) are contaminated.

Our results are the first SSP-based indications of a steepening of the low-mass end of the IMF with increasing galaxy mass within the class of LRG/ETGs. Our results (i) support a similar trend first found by Treu et al. (2010), (ii) extend the evidence based on SSP models that the IMF steepens from spiral to early-type galaxies (vDC10), and (iii) are in agreement with a similar trend found by Cappellari et al. (2012). The upper limit of $x \leqslant 3.0$, based on one of the most massive ETG systems in our sample, a gravitational lens, also supports our previous similar finding that extremely bottom-heavy IMFs are excluded (Spiniello et al. 2011). 


\section{Conclusions}

I presented the first results from the X-Shooter Lens Survey (XLENS) project which aims to study the internal structure and the mass profile of a sample of massive lens ETGs with $\sigma_{*}^{\text {ETG }} \geqslant 250 \mathrm{~km} / \mathrm{s}$ using X-Shooter. The final goal of the survey is to separate the luminous and dark matter in the internal regions of massive red, old galaxies in order to study the interaction between these components and their impact on galaxies assembly and evolution in the hierarchical galaxy formation framework. In this context, characterizing the slope of the IMF and assess whether it is universal and similar to the one of the Milky Way, or if it depends on galaxy mass, on epoch of formation or on local density, is critical. The combination of gravitational lensing, stellar kinematics and spectroscopic tracers of low-mass stars, as a part of the XLENS Survey, has proven to be a powerful tool to constrain the low-mass end of the IMF from galaxy spectra. Our first finding supports the idea of a non-universal IMF. We find that massive ETGs (i.e. $\sigma_{*}^{\text {ETG }} \geqslant$ 250) (i) have an IMF slope most consistent with Salpeter, excluding Chabrier/Kroupatype IMFs, (ii) show evidence in their optical line-indices (e.g. NaI and TiO2) that their IMF steepens mildly with increasing galaxy mass. By using lensing constraints on the total mass enclosed within the Einstein radius of the lens ETGs, we also find that the IMF slope most likely cannot exceed $x \approx 3$. A full spectral comparison extended to the NIR, where most of the features that show different strenght in M-dwarfs and in cool giants are present, together with new data and more flexible models, are necessary to further strengthen these results and completely break the degeneracy between IMF variations and variations of other SSP parameters such as age and metallicity.

The author would like to thank her supervisors L.V.E. Koopmans and S.C. Trager for their guidance, support, time and new ideas, and her collaborators for useful help and comments.

\section{References}

Auger, M. W., Treu, T., Bolton, A. S., et al. 2009, ApJ, 705, 1099

Auger, M. W., Treu, T., Gavazzi, R., et al. 2010, ApJL, 721, L163

Auger, M. W., Treu, T., Bolton, A. S., et al. 2010, ApJ, 724, 511

Barnabè, M., Czoske, O., Koopmans, L. V. E., et al. 2011, MNRAS, 415, 2215

Bastian, N., Covey, K. R., \& Meyer, M. R. 2010, ARAA, 48, 339

Bolton, A. S., Burles, S., Koopmans, L. V. E., et al. 2006, ApJ, 638, 703

Cappellari, M., McDermid, R. M., Alatalo, K., et al. 2012, Nature, 484, 485

Chabrier, G. 2003, PASP, 115, 763

Conroy, C. \& van Dokkum, P. 2012, ApJ, 747, 69

Davé, R. 2008, MNRAS, 385, 147

Dutton, A. A., Mendel, J. T., \& Simard, L. 2012, MNRAS, 422, L33

Kroupa, P. 2001, MNRAS, 322, 231

Graves, G. J., Faber, S. M., \& Schiavon, R. P. 2009, ApJ, 698, 1590

Napolitano, N. R., Romanowsky, A. J., \& Tortora, C. 2010, MNRAS, 405, 2351

Spiniello, C., Koopmans, L. V. E., Trager, S. C., Czoske, O., \& Treu, T. 2011, MNRAS, 417, 3000

Spiniello, C., Trager, S. C., Koopmans, L. V. E., \& Chen, Y. P. 2012, ApJL, 753, L32

Trager, S. C., Faber, S. M., Worthey, G., \& González, J. J. 2000, AJ, 120, 165

Treu, T., Auger, M. W., Koopmans, L. V. E., et al. 2010, ApJ, 709, 1195

van Dokkum, P. G. 2008, ApJ, 674, 29

van Dokkum, P. G. \& Conroy, C. 2010, Nature, 468, 940

Worthey, G. 1992, in: B. Barbuy, A. Renzini (eds.), IAU Symposium 149, The Stellar Populations of Galaxies, 149, 507 